\title{
The evidence of porcine hemagglutinating encephalomyelitis virus induced nonsuppurative encephalitis as the cause of death in piglets
}

Zi $\mathrm{Li}^{1}$, Wenqi He ${ }^{1}$, Yungang Lan ${ }^{1}$, Kui Zhao ${ }^{1}$, Xiaoling Lv ${ }^{1}$, Huijun Lu ${ }^{2}$, Ning Ding ${ }^{1}$, Jing Zhang ${ }^{1}$, Junchao Shi ${ }^{1}$, Changjian Shan ${ }^{1}{ }^{\text {, Feng Gao }}{ }^{\text {Corresp. }} 1$

${ }^{1}$ Key Laboratory of Zoonosis, Ministry of Education, College of Veterinary Medicine, Jilin University, Jilin, China

2 Key Laboratory of Zoonosis, Ministry of Education, Institute of Zoonosis, Jilin University, Jilin, China

Corresponding Author: Feng Gao

Email address: gaofeng@jlu.edu.cn

An acute outbreak of porcine hemagglutinating encephalomyelitis virus (PHEV) infection in piglets, characterized with neurological symptoms, vomiting, diarrhea, and wasting, occurred in China. Coronavirus-like particles were observed in the homogenized tissue suspensions of the brain of dead piglets by electron microscopy, and a wild PHEV strain was isolated, characterized, and designated as PHEV-CC14. Histopathologic examinations of the dead piglets showed characteristics of non-suppurative encephalitis, and some neurons in the cerebral cortex were degenerated and necrotic, and neuronophagia. Similarly, mice inoculated with PHEV-CC14 were found to have central nervous system (CNS) dysfunction, with symptoms of depression, arched waists, standing and vellicating front claws. Furthmore, PHEV-positive labeling of neurons in cortices of dead piglets and infected mice supported the viral infections of the nervous system. Then, the major structural genes of PHEV-CC14 were sequenced and phylogenetically analyzed, and the strain shared 95\%-99.2\% nt identity with the other PHEV strains available in GenBank. Phylogenetic analysis clearly proved that the wild strain clustered into a subclass with a HEV-JT06 strain. These findings suggested that the virus had a strong tropism for CNS, in this way, inducing nonsuppurative encephalitis as the cause of death in piglets.

Simultaneously, the predicted risk of widespread transmission showed a certain variation among the PHEV strains currently circulating around the world. Above all, the information presented in this study can not only provide good reference for the experimental diagnosis of PHEV infection for pig breeding, but also promote its new effective vaccine development. 
1 The evidence of porcine hemagglutinating encephalomyelitis

2 virus induced nonsuppurative encephalitis as the cause of

3 death in piglets

4 Zi Li ${ }^{1 *}$, Wenqi $\mathrm{He}^{1 *}$, Yungang Lan ${ }^{1}$, Kui Zhao ${ }^{1}$, Xiaoling $\mathrm{Lv}^{1}$, Huijun $\mathrm{Lu}^{2}$, Ning Ding ${ }^{1}$, Jing

5 Zhang ${ }^{1}$, Junchao Shi ${ }^{1}$, Changjian Shan ${ }^{1}$, Feng Gao ${ }^{1 \#}$

6

$7 \quad{ }^{1}$ Key Laboratory of Zoonosis, Ministry of Education, College of Veterinary Medicine, Jilin

8 University. Changchun, Jilin, China.

$9 \quad{ }^{2}$ Key Laboratory of Zoonosis, Ministry of Education, Institute of Zoonosis, Jilin University,

10 Changchun, Jilin, China.

Corresponding Author:

Feng Gao ${ }^{1}$

5333 Xi'an Road, Changchun, Jilin, 130062, China

Street Address, City, State/Province, Zip code, Country

Email address: gaofeng@jlu.edu.cn

*They contributed equally to this work. 


\section{Abstract}

An acute outbreak of porcine hemagglutinating encephalomyelitis virus (PHEV) infection in piglets, characterized with neurological symptoms, vomiting, diarrhea, and wasting, occurred in China. Coronavirus-like particles were observed in the homogenized tissue suspensions of the brain of dead piglets by electron microscopy, and a wild PHEV strain was isolated, characterized, and designated as PHEV-CC14. Histopathologic examinations of the dead piglets showed characteristics of non-suppurative encephalitis, and some neurons in the cerebral cortex were degenerated and necrotic, and neuronophagia. Similarly, mice inoculated with PHEV-CC14 were found to have central nervous system (CNS) dysfunction, with symptoms of depression, arched waists, standing and vellicating front claws. Furthmore, PHEV-positive labeling of neurons in cortices of dead piglets and infected mice supported the viral infections of the nervous system. Then, the major structural genes of PHEV-CC14 were sequenced and phylogenetically analyzed, and the strain shared 95\%-99.2\% nt identity with the other PHEV strains available in GenBank. Phylogenetic analysis clearly proved that the wild strain clustered into a subclass with a HEVJT06 strain. These findings suggested that the virus had a strong tropism for CNS, in this way, inducing nonsuppurative encephalitis as the cause of death in piglets. Simultaneously, the predicted risk of widespread transmission showed a certain variation among the PHEV strains currently circulating around the world. Above all, the information presented in this study can not only provide good reference for the experimental diagnosis of PHEV infection for pig breeding, but also promote its new effective vaccine development. 
Porcine hemagglutinating encephalomyelitis virus (PHEV) belongs to the order Nidovirales,

42 family Coronaviridae, and genus Coronavirus, and causes encephalomyelitis or vomiting and

43 wasting disease in suckling piglets (Andries \& Pensaert 1981; Mengeling et al. 1972). Previous

44 studies have demonstrated that the virus has a strong tropism for the upper respiratory tract and is

45 propagated through the neural route (Andries \& Pensaert 1980). The disease caused by PHEV

46 was first reported in Canada in 1958 (Roe \& Alexander 1958), and the pathogen was first

47 isolated from the brains of suckling piglets with encephalomyelitis in 1962 (Greig et al. 1962).

48 Since then, the infection has been reported in the United States, Japan, Argentina, Belgium,

49 South Korea, China and other pig-raising countries (Gao et al. 2011; Hirano \& Ono 1998;

50 Pensaert \& Callebaut 1974; Quiroga et al. 2008; Rho et al. 2011; Sasseville et al. 2001). Today,

51 many serological surveys have revealed that PHEV is widespread, and there are frequent

52 subclinical infections ( $\mathrm{Li}$ et al. 2013).

In China, PHEV infection first occurred in Beijing in 1985, and the outbreaks caused

54 enormous economic losses for the pig industry (Chen et al. 2012; Dong et al. 2014; Gao et al.

55 2011). Here we report that there is a suspected outbreak of PHEV infection on a farm in

56 Changchun of Jilin Province, in 2014, resulting in serious economic losses. Many infected

57 piglets characterized with vomiting and nerve symptoms, and some cases were accompanied by

58 screaming or diarrhea; all of the piglets with clinical symptoms died finally. In this paper, the

59 diagnosis was made on the basis of pathologic features, immunohistochemistry, microbiological

60 detection, and RT-PCR. A PHEV field strain was isolated from the brain tissue of infected 
61 piglets, and the major structural proteins of the strain were sequenced to identify genetic

62 relationships with other coronaviruses of the genus Betacoronavirus. 


\section{Materials \& Methods}

\section{Sample collection and testing}

65

On March 2014, there was an acute outbreak of suspected porcine hemagglutinating encephalomyelitis in suckling pigs on a farm with a total of 502 sows in Changchun, Jilin province, China. At the time of the outbreak, these pigs had not been immunized with any PHEV vaccines; the total proportion of deaths in piglets that had not been weaned was 46.7\% (140 dead piglets). The collected samples were tested for PHEV using real-time reverse transcriptionpolymerase chain reaction (RT-PCR) targeting the HE gene, as well as for other viruses that cause similar clinical symptoms among swine, including porcine epidemic diarrhea virus (PEDV), porcine transmissible gastroenteritis virus (TGEV), porcine deltacoronavirus (PDCoV), and pseudorabies virus (PRV). All experiments on piglets research were performed in accordance with Animal Welfare Ethical Committee of Jilin University guidelines and regulations (permission number 2012-CVM-12). The involved RT-PCR primers were designed based on the most conserved segment of their genomes (Table 1), and subsequently validated by BLAST (http://www.ncbi.nlm.nih.gov/BLAST) with sequences from GenBank. The original samples were diluted 10-fold with phosphate-buffered saline (PBS) and were centrifuged at $3,000 \times \mathrm{g}$ at $4^{\circ} \mathrm{C}$ for $10 \mathrm{~min}$. The supernatant was filtered through a $0.22-\mu \mathrm{m}$ syringe filter, and was used as inoculums for BALB/c mice or for virus isolation in Neuro-2a cell culture.

\section{Histopathologic examination}


Postmortem examinations were performed and samples submitted for histopathologic

83 examination, including tissues from the brain, heart, spleen, liver, kidneys, and lungs. Paraffinembedded sections of brain that had characteristic microscopic lesions were examined by hematoxylin-eosin staining. Selected paraffin sections for PHEV antigen detection by immunohistochemistry (IHC) tests were treated with normal goat serum for 1 hour and anti-HEV $67 \mathrm{~N}$ monoclonal antibody (Chen et al. 2012) (diluted 1:100) overnight; then, the staining procedure was performed according to the kit instructions.

\section{Inoculation of BALB/c mice with PHEV strains}

Thirty 3-week-old male BALB/c mice were randomly and equally divided into 3 groups.

The mice in group 1 were inoculated with the original filtered brain tissue by the intranasal route, the mice in group 2 were inoculated with HEV 67N (GenBank: AY048917) in the same manner, and the mice in the third group formed a negative control group. The permission to work with laboratory animals was obtained from the Animal Welfare Ethical Committee of the College of Veterinary Medicine, Jilin University, China (permission number 2012-CVM-12). All of the mice experiments were carried out at Bio-Safety Level 2 (BSL-2) facilities at the Key Laboratory of Zoonosis, Ministry of Education, College of Veterinary Medicine, Jilin University. Clinical signs were monitored, and immunofluorescence assay (IFA) was performed. The PHEV monoclonal antibody (diluted 1:500) was used as the primary antibody, and a 1:200 dilution of affinity purified fluorescein-labeled goat anti-mouse IgG was used as the second antibody. Cell staining was examined using a fluorescence microscope. 
102

103

104

105

106

107

108

109

110

111

112

113

114

115

\section{Virus isolation and propagation}

The Neuro-2a cell line was used to isolate PHEV from the original field and from mousepassaged PHEV samples. Cultured cells were propagated in Dulbecco's Modified Eagle Medium (DMEM, Gibco, USA) supplemented with 10\% heat-inactivated fetal bovine serum (Hyclone, Logan, UT, USA) and 1\% antibiotic-antimycotic (Gibco, USA). Briefly, a monolayer of cells was washed twice with 2\% DMEM, and then was inoculated with the filtered samples. After adsorption for $1 \mathrm{~h}$ at $37^{\circ} \mathrm{C}$ in $5 \% \mathrm{CO}_{2}$, the cells were washed 3 times, and $2 \%$ DMEM was added. The cell cultures were examined daily for cytopathic effect (CPE). When more than $80 \% \mathrm{CPE}$ was evident in the inoculated cell monolayers, the cells and supernatants were harvested together and used as seed stocks for the next passage. After serial passage, the cell cultures were clarified by centrifugation at $3,000 \times \mathrm{g}$ for $30 \mathrm{~min}$ at $4^{\circ} \mathrm{C}$ and then were further ultracentrifuged at 20,000

$\times \mathrm{g}$ for $2 \mathrm{~h}$ at $4^{\circ} \mathrm{C}$ using an ultracentrifuge. The pellet was resuspended and submitted for virological investigation using electron microscopy (EM). The virus isolate was designated PHEV-CC14.

\section{Virus titration and purification by plaque assay}

The $100 \%$ confluent Neuro-2a cells in 6-well plates were used for plaque assays of PHEVCC14 propagation and purification. Briefly, the wells were inoculated with 10-fold serially diluted virus $\left(0.2 \mathrm{~mL} /\right.$ well), followed by adsorption for $1 \mathrm{~h}$ at $37^{\circ} \mathrm{C}$ in $5 \% \mathrm{CO}_{2}$; then, the wells were washed 3 times, and $2 \mathrm{~mL}$ of the agarose/MEM mixture (1:1) were added. After the plaques were counted and confirmed, uniform and clear plaques were chosen to inoculate 6-well plates 
122

123

124

125

directly. When CPE was observed, the positive clones were harvested, and the viral titers were determined. When the Neuro-2a cells were confluent in 96 -well plates, $100 \mathrm{~mL}$ of 10 -fold dilutions of the purified virus were absorbed for $1 \mathrm{~h}$. Viral CPE was monitored for 5 to 7 days, and virus titers were determined by $50 \%$ tissue culture infectious dose $\left(\mathrm{TCID}_{50}\right)$.

\section{PHEV-CC14 structural gene sequencing and phylogenetic analysis}

All five of the main structural protein genes, hemagglutinin-esterase (HE), spike (S), small membrane (E), membrane (M) and nucleocapsid $(\mathrm{N})$, of PHEV in the original specimen, in the $\mathrm{BALB} / \mathrm{c}$ mice infected with passaged PHEV-CC14, and in cell culture were amplified, cloned and sequenced. All of the primers were designed according the sequence of the HEV 67N genome. Viral RNA was extracted from the brain tissue suspensions of symptomatic piglets using a commercial kit (QIAGEN, Germany), and the RNA was quantified using a spectrophotometer (BIO-RAD, USA). The RNA was converted to cDNA by an oligo (dT)priming strategy, and the genes were amplified using PrimeSTAR MAX DNA Polymerase (Takara, Japan). The purified PCR products were cloned into the pMD18-T vector (TaKaRa, Japan) and were introduced into E. coli DH5a by transformation. The recombinant plasmids were extracted and verified by PCR and then were sequenced at Shanghai Sangon Biological Engineering Technology and Services Co., Ltd. (China).

\section{The sequence data were assembled and analyzed using DNASTAR and NCBI BLAST} (http://blast.ncbi.nlm.nih.gov/Blast.cgi). The percentage similarities of the nucleotides and amino acids were analyzed using DNAMAN and DNASTAR software. The structural gene sequences 
142 and other coronavirus strains sequence were subjected to phylogenetic analysis using the

143 neighbor-joining method in MEGA software, version 6.06.

\section{Statistical analysis}

145 Statistical analysis was performed with either Student's t-test or one-way ANOVA with a

146 Bonferroni post hoc test with software provided by GraphPad Prism version 5. Data were

147 presented as means \pm S.E.M. $P$ values of $<0.05$ were considered statistically significant. 
148

149

150

151

152

153

154

155

156

157

158

159

160

161

162

163

164

165

166

167

Results

\section{Pathological examination and pathogen detection}

Clinical signs of these suspected infected suckling piglets were consisted of vomiting, diarrhea, wasting, dullness, screaming, anorexia, trembling, and ataxia (Fig.1A). Pathological examination showed that the main changes in the piglets were congestion, edema and hemorrhage in brain tissue (Fig.1B). None significant histopathological changes were found in other substantive organs. A total of 54 homogenized tissue suspensions of the brain, spinal cord, lungs, kidneys, spleen and intestinal contents from 9 suspected piglets were tested for PHEV by RT-PCR. Of these tested samples, eight of nine brain samples from young nursing pigs on the farms were PHEV positive, as well as eight of nine spinal cord samples and four of nine intestinal content samples (Table 2). Of the 20 PHEV-positive samples, all were negative for PEDV, TGEV, PDCoV, and PRV.

\section{Histopathologic examination of the PHEV-infected piglets}

Postmortem examinations were performed on 7 infected piglets for pathologic evaluation.

Samples submitted for histopathologic examination included brains from PHEV-infected piglets and antigen-negative piglets. Microscopic examination of brain samples showed characteristics of non-suppurative encephalitis. A large number of glial cells were aggregated to glial nodules in the infected brains (Fig.2A and 2B). Neurons in the cerebral cortex were degenerated and necrotic, and neuronophagia was widespread (Fig.2C and 2D). Selected paraffin sections of brain samples that had characteristic microscopic lesions were examined for PHEV antigen by IHC 
168

169

170

tests with an anti-PHEV monoclonal antibody. In the brains, antigen-positivity in the cytoplasm of nerve cells was distributed widely in the cortical neurons (Fig.2E). Brain samples from the healthy pig were normal (Fig.2F).

\section{Pathogenicity of HEV 67N and PHEV-CC14 in BALB/c mice}

Mice in two infected groups were inoculated with HEV 67N and PHEV-CC14, respectively, and were monitored daily for clinical signs of disease. Mice in the HEV 67N-infected group showed typical neurological damage, with symptoms of depression, arched waists, standing and vellicating front claws at 3 days post-inoculation (dpi). The same symptoms occurred in the PHEV-CC14-infected group (Fig.3A and 3B), but the emergence time was slightly delayed (Fig.3C, $\mathrm{P}<0.05$ ). All of the infected mice died within a week, and the mice in the control group survived normally. Paraffin-embedded sections of the infected mouse brain samples were positive for PHEV in the cytoplasm of nerve cells by IFA using a mouse anti-PHEV monoclonal antibody. In the brain, antigen-positive neurons were distributed widely in the cerebral cortex and hippocampus (Fig.4). In the cerebellum, viral-specific antigen was detected in the Purkinje cells (Fig.4) but in only a few granular cells.

\section{Isolation and purification of PHEV-CC14 strain}

The Neuro-2a cell monolayer was inoculated with original field and mouse-passaged PHEVpositive samples. At $3 \mathrm{dpi}$, the inoculated cell monolayer showed visible $\mathrm{CPE}$, in the form of gathering pyknosis and rounded cells that rapidly detached from the monolayer on 4 dpi (Fig.5B), the mock-inoculated Neuro-2a cells showing normal cells (Fig.5A). The virus was further 
188 189 190

serially passed in Neuro-2a cells for a total of 18 passages. Virus growth was confirmed by IFA using the antiserum PHEV, and the antigens were mostly located in the cytoplasm (Fig.5C). To confirm PHEV replication, viral RNA was extracted from the culture supernatants and was tested by RT-PCR. The presence of PHEV particles in the infected cells was also examined by EM. The EM results showed multiple virus particles approximately 110 to $130 \mathrm{~nm}$ in diameter with typical coronavirus morphology (Fig.5D). Thus, the PHEV strain was successfully isolated and was designated as PHEV-CC14.

Plaque assay was used to plaque isolates and to purify PHEV on Neuro-2a cells, and large clear plaques were evident under an agar overlay medium on the cells. The cloned virus PHEVCC14 was tested by RT-PCR and was further serially passaged to 20 passages on Neuro-2a cells (5.2 $\left.\log _{10} \mathrm{PFU} / \mathrm{mL}\right)$. During the serial passages, significant increases in viral RNA titers were observed following each cell passage. The infectious titers of PHEV-CC14 were determined by $\mathrm{TCID}_{50}$ and were calculated according to the Reed-Muench method. As shown in Figure 6, there were no significant differences in replication or proliferation between the PHEV-CC14 and HEV-67N strains in the Neuro-2a cells, but the RNA virus titers in the PHEV-CC14-infected cells $\left(10^{6.03} \mathrm{TCID}_{50} / \mathrm{mL}\right)$ were slightly higher than those in the HEV $67 \mathrm{~N}$-infected cells $\left(10^{5.43} \mathrm{TCID}_{50} / \mathrm{mL}\right)$ after $72 \mathrm{~h}$ post-inoculation.

\section{Sequence and phylogenetic analysis}

To examine whether genetic changes occurred in the PHEV-CC14 strain (GenBank:

KU127229) compared with other PHEVs available in GenBank, the major structure genes were 
208 amplified by specific primers (Table 3) and sequenced. A total of 8,123 nucleotides were

209 determined for strain PHEV-CC14, covering five complete structure genes -- HE, S, E, M and N

$210-$ and the locations of the organization of the targeted genes were sketched in a conceptual map

211 (Fig.7A). Therewith, the corresponding nucleotides and deduced amino acid sequences of the

212 PHEV-CC14 strain were compared with the homologous sequences of PHEVs. The results

213 showed that the PHEV-CC14 strain shared 95\%-99.2\% nt identities with the other PHEV strains

214 available in GenBank. The structural genes of the PHEV-CC14 strain had the greatest nucleotide

215 sequence similarity (99.2\%) to the HEV-JT06 strain (GenBank: ED919227.1), and it shared 99\%

216 with HEV 67N (GenBank: AY078417.1). Compared with the HEV 67N strain, there were four

217 nucleotide sense mutations at positions 12 and 114 in the HE gene, 381 in the S gene, 146 in the

$218 \mathrm{M}$ gene. These nucleotide changes all induced corresponding amino acid (aa) changes (S12G and

219 T114I in the HE protein; R381H in the S protein; A146T in the M protein). However, residues in

220 the E and N genes of PHEV-CC14 strains were highly conserved in identity with other PHEV

221 reference strains in the GenBank database.

A phylogenetic tree was constructed using the five genes (HE, S, E, M, and N) of PHEV-

CC14 with some other PHEV strains obtained from GenBank Database, as well as several members of the coronaviruses (Fig.7B). Phylogenetic analysis of the five genes clearly showed that the PHEV-CC14 strain clustered into a subclass with a HEV-JT06 strain from China isolated in 2006, and a similar finding showed that the PHEV stains in China were highly homologous 227 with a North American strain (AY078417). Additionally, the homology of the deduced amino 
228 acid sequences between the PHEV-CC14 strain and HCoV-OC43 (GenBank: KF530085) was as 229 high as $91 \%$. 


\section{Discussion}

In March 2014, there was a suspected outbreak of PHEV infection on a farm in Changchun

232

233

234

235

236

237

238

239

240

241

242

243

244

245

246

247

248

249

in the Jilin province of China. The clinical signs consisted of vomiting, twitching, wasting, and diarrhea were observed in suckling piglets. The histologic autopsy showed that there were pinpoint petechiae in the kidneys, thinning of the intestinal wall, and hemorrhage in the brain.

Due to the great similarity between PHEV and pseudorabies virus (PRV) infection in piglets, it was difficult to distinguish them only by clinical and autopsy symptoms. Therefore, PRV and PHEV were first detected by PCR and RT-PCR, respectively, and the test results showed that PRV was negative, and PHEV was positive, thus excluding PRV infection. At the same time, no sow abortions or stillbirth phenomena were found in the pigs, also supporting the above results. Immunohistochemical staining results further confirmed PHEV infection in the brains of the dead piglets. Vomiting and neurological symptoms are common in piglets infected with PHEV, but the symptom of diarrhea is relatively rare. Because the outbreak was characterized by vomiting and diarrhea, some other viral infections with similar clinical symptoms have been reported in pigs, including PEDV, TGEV and DPCoV (Ma et al. 2015; Song et al. 2015; Tanaka et al. 2015). In this study, these viruses were further detected to exclude misdiagnosis or mixed infection. Thus, the case was identified as simple PHEV infection, and we successfully isolated a PHEV field strain as PHEV-CC14.

PHEV has a typical neural tropism, and it invades the central nervous system via the peripheral nervous system (Hirano et al. 2004; Lafaille et al. 2015; Lee et al. 2011; Zhang et al. 
250 2014). Previous studies have shown that the virus successfully killed 1 - to 8-week-old mice

251 readily by different routes, and viral antigen was detected in both peripheral nerves and the CNS

252 (Hirano et al. 2001; Hirano et al. 1995; Hirano et al. 2006). In this paper, three-week-old

$253 \mathrm{BALB} / \mathrm{c}$ mice were chosen for inoculation with PHEV-CC14 by the intranasal route, and the

254 results of the experiment confirmed that PHEV-CC14 had strong pathogenicity to mice.

Viral titers of PHEV-CC14 or HEV 67N were determined by TCID $_{50}$, and the growth curve showed that there was no significant difference in replication or proliferation between them. To characterize the virus isolates, the complete structural genes were sequenced and analyzed, and the phylogenetic relationships among the coronavirus strains were determined. Phylogenetic trees showed that the wild type and HEV-JT06 shared the highest homology, and that identified with HEV $67 \mathrm{~N}$ was $99 \%$. These findings suggested that PHEV strains currently circulating in China are closely related. Notably, the PHEV strain JT06 isolated from Jilin province, China, in 2006 was most closely related to the emerging PHEV-CC14 strains, suggesting that they could be derived from a similar ancestral strain. Furthermore, we performed sequence alignment and homology analysis between PHEV-CC14 and other coronaviruses, which including MHV, $\mathrm{BCoV}, \mathrm{HCoV}-\mathrm{OC} 43$ and Bat $\mathrm{CoV}$, and we found it shared up to $91 \%$ homology with $\mathrm{HCoV}$ OC43 (GenBank: KF530085) (Gonzalez et al. 2003; Li 2015; Snijder et al. 1993). This finding suggested that, although PHEV infection in humans has not been reported currently, there is a definite potential threat to human health. 
According to the deduced amino acids of the PHEV-CC14 strains, genetic evolution and

variation analyses were performed. It was found that the broad variation occurred in the encoded

structural proteins and functional region. There were five major structural proteins of PHEV, HE,

S, E, M and N proteins, encoded from 5'UTR to 3'UTR (Vijgen et al. 2006; Weiss \& Navas-

Martin 2005). Sequence analyses of the five structural proteins of the PHEV-CC14 field isolate

et al. 1990; Vieler et al. 1995). The HE proteins of some coronaviruses are involved in the

release of virions from the host cell, and it has been shown to have acetylesterase activity and to

function as a receptor-destroying enzyme, which might be related to the early adsorption of

coronavirus (Schultze et al. 1991). Compared with HEV 67N, there were two amino acids (S12G,

T114I) in the HE protein of PHEV-CC14 that were meaningful mutations, while six amino acid

variations were observed (S12G, S15G, K49N, T114I, V116A, L161F) when blasted with

the IAF-404 strain. We hypothesize that the amino acid variation of the HE protein might have a

certain effect on replication and virulence, but it was difficult to explain the differences in

virulence among the pigs, based on the amino acid changes. In addition, there were some

variations of the amino acids in the S protein, which plays vital roles in viral entry, cell-to-cell

spread, and the determination of tissue tropism (Dong et al. 2015; Lu et al. 2015). Therefore, the

differences in virulence of PHEV strains might be caused by multiple factors, and the variation

of the whole genome has resulted in changes in their antigenic differences.

\section{Conclusions}



significance.

\section{References}

Andries K, and Pensaert M. 1981. Vomiting and wasting disease, a coronavirus infection of pigs. Advances in experimental medicine and biology 142:399-408.

Andries K, and Pensaert MB. 1980. Immunofluorescence studies on the pathogenesis of 
309

310

311

312

313

314

315

316

317

318

319

320

321

322

323

324

325

326

327

328

329

Identification and genetic characterization of porcine hemagglutinating encephalomyelitis virus from domestic piglets in China. Archives of virology 159:2329-2337. 10.1007/s00705014-2070-y

Gao W, Zhao K, Zhao C, Du C, Ren W, Song D, Lu H, Chen K, Li Z, Lan Y, Xie S, He W, and Gao F. 2011. Vomiting and wasting disease associated with hemagglutinating encephalomyelitis viruses infection in piglets in Jilin, China. Virology journal 8:130.

$10.1186 / 1743-422 X-8-130$

Gonzalez JM, Gomez-Puertas P, Cavanagh D, Gorbalenya AE, and Enjuanes L. 2003. A comparative sequence analysis to revise the current taxonomy of the family Coronaviridae. Archives of virology 148:2207-2235. 10.1007/s00705-003-0162-1

Greig AS, Mitchell D, Corner AH, Bannister GL, Meads EB, and Julian RJ. 1962. A Hemagglutinating Virus Producing Encephalomyelitis in Baby Pigs. Canadian journal of comparative medicine and veterinary science 26:49-56.

Hirano N, Haga S, Sada Y, and Tohyama K. 2001. Susceptibility of rats of different ages to inoculation with swine haemagglutinating encephalomyelitis virus (a coronavirus) by various routes. Journal of comparative pathology 125:8-14. 10.1053/jcpa.2001.0471

Hirano N, Nomura R, Tawara T, Ono K, and Iwasaki Y. 1995. Neuronal spread of swine hemagglutinating encephalomyelitis virus (HEV) $67 \mathrm{~N}$ strain in 4-week-old rats. Advances in experimental medicine and biology 380:117-119.

Hirano N, Nomura R, Tawara T, and Tohyama K. 2004. Neurotropism of swine haemagglutinating encephalomyelitis virus (coronavirus) in mice depending upon host age 
and route of infection. Journal of comparative pathology 130:58-65.

331

332

Hirano N, and Ono K. 1998. A serological survey of human coronavirus in pigs of the Tohoku District of Japan. Advances in experimental medicine and biology 440:491-494.

Hirano N, Taira H, Sato S, Hashikawa T, and Tohyama K. 2006. Antibody-mediated virus clearance from neurons of rats infected with hemagglutinating encephalomyelitis virus. Advances in experimental medicine and biology 581:391-394. 10.1007/978-0-387-33012$9+69$

Lafaille FG, Ciancanelli MJ, Studer L, Smith G, Notarangelo L, Casanova JL, and Zhang SY. 2015. Deciphering Human Cell-Autonomous Anti-HSV-1 Immunity in the Central Nervous System. Frontiers in immunology 6:208. 10.3389/fimmu.2015.00208

Lee H, Sunden Y, Ochiai K, and Umemura T. 2011. Experimental intracerebral vaccination protects mouse from a neurotropic virus by attracting antibody secreting cells to the CNS. Immunology letters 139:102-109. 10.1016/j.imlet.2011.05.008

Li F. 2015. Receptor recognition mechanisms of coronaviruses: a decade of structural studies. Journal of virology 89:1954-1964. 10.1128/JVI.02615-14

Li YC, Bai WZ, Hirano N, Hayashida T, Taniguchi T, Sugita Y, Tohyama K, and Hashikawa T. 2013. Neurotropic virus tracing suggests a membranous-coating-mediated mechanism for transsynaptic communication. The Journal of comparative neurology 521:203-212. $10.1002 /$ cne. 23171

Lu G, Wang Q, and Gao GF. 2015. Bat-to-human: spike features determining 'host jump' of coronaviruses SARS-CoV, MERS-CoV, and beyond. Trends in microbiology 23:468-478. 
351

352

353

354

355

356

357

358

359

360

361

362

363

364

365

366

367

368

369

370

371

10.1016/j.tim.2015.06.003

Ma Y, Zhang Y, Liang X, Lou F, Oglesbee M, Krakowka S, and Li J. 2015. Origin, evolution, and virulence of porcine deltacoronaviruses in the United States. mBio 6:e00064.

\subsection{8/mBio.00064-15}

Mengeling WL, Boothe AD, and Ritchie AE. 1972. Characteristics of a coronavirus (strain 67N) of pigs. American journal of veterinary research 33:297-308.

Pensaert MB, and Callebaut PE. 1974. Characteristics of a coronavirus causing vomition and wasting in pigs. Archiv fur die gesamte Virusforschung 44:35-50.

Quiroga MA, Cappuccio J, Pineyro P, Basso W, More G, Kienast M, Schonfeld S, Cancer JL, Arauz S, Pintos ME, Nanni M, Machuca M, Hirano N, and Perfumo CJ. 2008.

Hemagglutinating encephalomyelitis coronavirus infection in pigs, Argentina. Emerg Infect Dis 14:484-486. 10.3201/eid1403.070825

Rho S, Moon HJ, Park SJ, Kim HK, Keum HO, Han JY, Van Nguyen G, and Park BK. 2011. Detection and genetic analysis of porcine hemagglutinating encephalomyelitis virus in South Korea. Virus genes 42:90-96. 10.1007/s11262-010-0551-y

Roe CK, and Alexander TJ. 1958. A Disease of Nursing Pigs Previously Unreported in Ontario. Canadian journal of comparative medicine and veterinary science 22:305-307.

Sasseville AM, Gelinas AM, Sawyer N, Boutin M, and Dea S. 2001. Biological and molecular characteristics of an HEV isolate associated with recent acute outbreaks of encephalomyelitis in Quebec pig farms. Advances in experimental medicine and biology 494:57-62.

Schultze B, Hess G, Rott R, Klenk HD, and Herrler G. 1990. Isolation and characterization of the 
372

373

374

375

376

377

378

379

380

381

382

383

384

385

386

387

388

389

390

391

392

acetylesterase of hemagglutinating encephalomyelitis virus (HEV). Advances in experimental medicine and biology 276:109-113.

Schultze B, Wahn K, Klenk HD, and Herrler G. 1991. Isolated HE-protein from hemagglutinating encephalomyelitis virus and bovine coronavirus has receptor-destroying and receptor-binding activity. Virology 180:221-228.

Snijder EJ, Horzinek MC, and Spaan WJ. 1993. The coronaviruslike superfamily. Advances in experimental medicine and biology 342:235-244.

Song D, Moon H, and Kang B. 2015. Porcine epidemic diarrhea: a review of current epidemiology and available vaccines. Clinical and experimental vaccine research 4:166-176. 10.7774/cevr.2015.4.2.166

Tanaka Y, Sasaki T, Matsuda R, Uematsu Y, and Yamaguchi T. 2015. Molecular epidemiological study of feline coronavirus strains in Japan using RT-PCR targeting nsp14 gene. BMC veterinary research 11:57. 10.1186/s12917-015-0372-2

Vieler E, Schlapp T, Anders C, and Herbst W. 1995. Genomic relationship of porcine hemagglutinating encephalomyelitis virus to bovine coronavirus and human coronavirus OC43 as studied by the use of bovine coronavirus S gene-specific probes. Archives of virology 140:1215-1223.

Vijgen L, Keyaerts E, Lemey P, Maes P, Van Reeth K, Nauwynck H, Pensaert M, and Van Ranst M. 2006. Evolutionary history of the closely related group 2 coronaviruses: porcine hemagglutinating encephalomyelitis virus, bovine coronavirus, and human coronavirus OC43. Journal of virology 80:7270-7274. 10.1128/JVI.02675-05 
393 Weiss SR, and Navas-Martin S. 2005. Coronavirus pathogenesis and the emerging pathogen

394 severe acute respiratory syndrome coronavirus. Microbiology and molecular biology reviews :

$395 \quad$ MMBR 69:635-664. 10.1128/MMBR.69.4.635-664.2005

396 Zhang L, Xu MM, Zeng L, Liu S, Liu X, Wang X, Li D, Huang RZ, Zhao LB, Zhan QL, Zhu D,

397 Zhang YY, Xu P, and Xie P. 2014. Evidence for Borna disease virus infection in

398 neuropsychiatric patients in three western China provinces. European journal of clinical

399 microbiology \& infectious diseases : official publication of the European Society of Clinical

$400 \quad$ Microbiology 33:621-627. 10.1007/s10096-013-1996-4 
402

403

404

405

406

407

408

409

410

411

412

413

414

415

416

417

418

419

420

421

\section{Legends}

Fig.1 Macropathologic images of PHEV infection in piglets from a farm in China. (A) The dead piglets with vomiting, neurologic symptoms, wasting, and diarrhea. (B) Pathologic autopsy showed congestion or hemorrhage in brain tissue.

Fig.2 Samples submitted for histopathologic examination by hematoxylin-eosin staining and IHC assay. (A) A large number of glial cells aggregating in the infected brains formed glial nodules (arrow); hematoxylin-eosin stain, x100. A boxed inset in the lower-right panel is shown at greater magnification in the image. (B) Brain samples in the healthy pig were normal; hematoxylin-eosin stain, x400. (C) The PHEV infected neurons showed shrunken, karyopyknosis and deeply stained (arrow); hematoxylin-eosin stain, x400. (D) Neurons in brain of healthy pig were well-arranged and distributed evenly and orderly; hematoxylin-eosin stain, x400. (E) Brains from an infected piglet showing PHEV-positive labeling in the cytoplasm of nerve cells (arrows); immunohistochemical staining, x400. (F) No PHEV-positive labeling of neurons in the negative control group; immunohistochemical staining, $\mathrm{x} 400$.

Fig.3 Mice experimentally infected with PHEV-CC14. (A) PHEV-CC14-infected mice showed arched waists, standing and vellicating front claws after at 3 dpi. (B) Mice in the control group survived normally. (C) Survival curves of BALB/c mice. $n=10$ mice per group; three independent experiments.

Fig.4 Visualization of PHEV-CC14-infected brains from BALB/c mice by immunofluorescent assay using PHEV monoclonal antibody (diluted 1:500). Immunofluorescent assay in the 
422

423

424

425

426

427 showing rounded and clustered cells, $\times 200$. (C) Detection of PHEV isolate in Neuro-2a cells by

428 immunofluorescent staining using PHEV monoclonal antibody (diluted 1:500), showing

429 immunofluorescent-positive staining mainly evident in the cytoplasm of infected cells (red),

430

431 432 picture represents $100 \mathrm{~nm}$ in length.

433

434

435

436

437

438

439

440

441

cerebral cortex, showing large numbers of PHEV-positive neurons (red); original magnification,

$\times 100$. PHEV-positive Purkinje cells of the cerebellum were distributed widely (red); original magnification, $\times 400$.

Fig.5 Isolation and propagation of PHEV in Neuro-2a cells. (A) Mock-inoculated Neuro-2a cells showing normal cells at 4 dpi, $\times 200$. (B) CPE of PHEV-CC14-inoculated Neuro-2a cells at 4 dpi, $\times 400$. (D) EM of PHEV-CC14-inoculated Neuro-2a cells. Crown-shaped spikes are visible. The samples were negatively stained with 3\% phosphotungstic acid. The magnification bar in the

Fig. 6 The growth curves of PHEV strains. Neuro-2a cells were, respectively, inoculated with PHEV-CC14 and HEV $67 \mathrm{~N}$. The $\mathrm{TCID}_{50}$ was measured at different time points, and the growth curves were plotted. There was no significant difference in replication or proliferation between the PHEV-CC14 strain and HEV-67N strain ( $\mathrm{p}>0.05)$.

Fig.7 Protein structure prediction and phylogenetic analysis. (A) Schematic illustration of the organization of the targeted genes coding for the five structural proteins, consisting of PHEV HE, S, E, M and N genes (reference virus HEV-67N). (B) Phylogenetic analyses based on amino acid sequences of the five major structural proteins from PHEV in this study (indicated with triangle) and other published PHEV sequences, as well as related coronaviruses. Reference sequences 
442 obtained from GenBank are indicated by strain names and accession numbers. The trees were

443 constructed using the neighbor-joining method in MEGA software, version 6.06. Bootstrap 444 analysis was performed on 1,000 trials, and values are indicated adjacent to the branching points.

445 
Figure $\mathbf{1}$ (on next page)

Macropathologic images of PHEV infection in piglets from a farm in China

Fig.1 Macropathologic images of PHEV infection in piglets from a farm in China. (A) The dead piglets with vomiting, neurologic symptoms, wasting, and diarrhea. (B) Pathologic autopsy showed congestion or hemorrhage in brain tissue. 
PeerJ

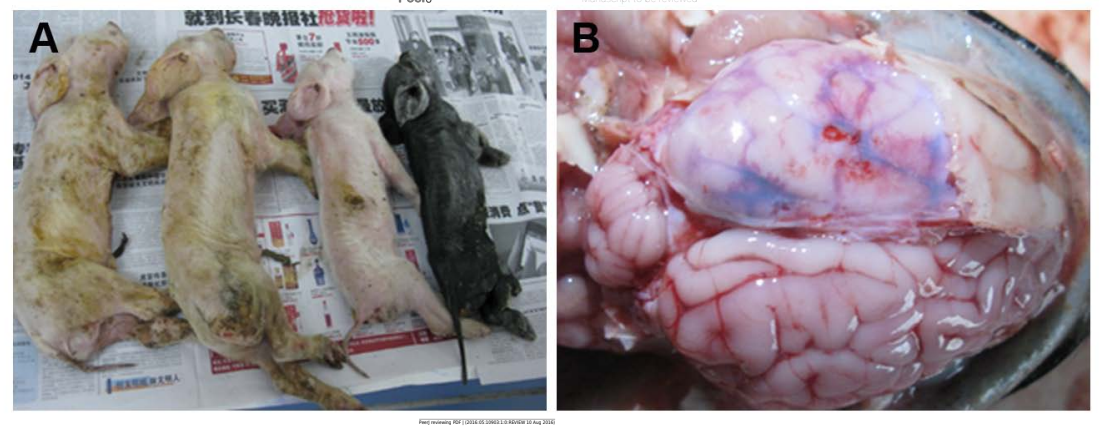




\section{Figure 2 (on next page)}

Samples submitted for histopathologic examination by hematoxylin-eosin staining and IHC assay

Fig.2 Samples submitted for histopathologic examination by hematoxylin-eosin staining and IHC assay. (A) A large number of glial cells aggregating in the affected brains formed glial nodules (arrow); hematoxylin-eosin stain, $x 100$. (B) Brain samples in the control group were normal; hematoxylin-eosin stain, $x 400$. (C) Brains from an affected piglet showing PHEVpositive labeling in the cytoplasm of nerve cells (arrows); immunohistochemical staining, $x 400$. (D) No PHEV-positive labeling of neurons in the negative control group; immunohistochemical staining, $x 400$. 


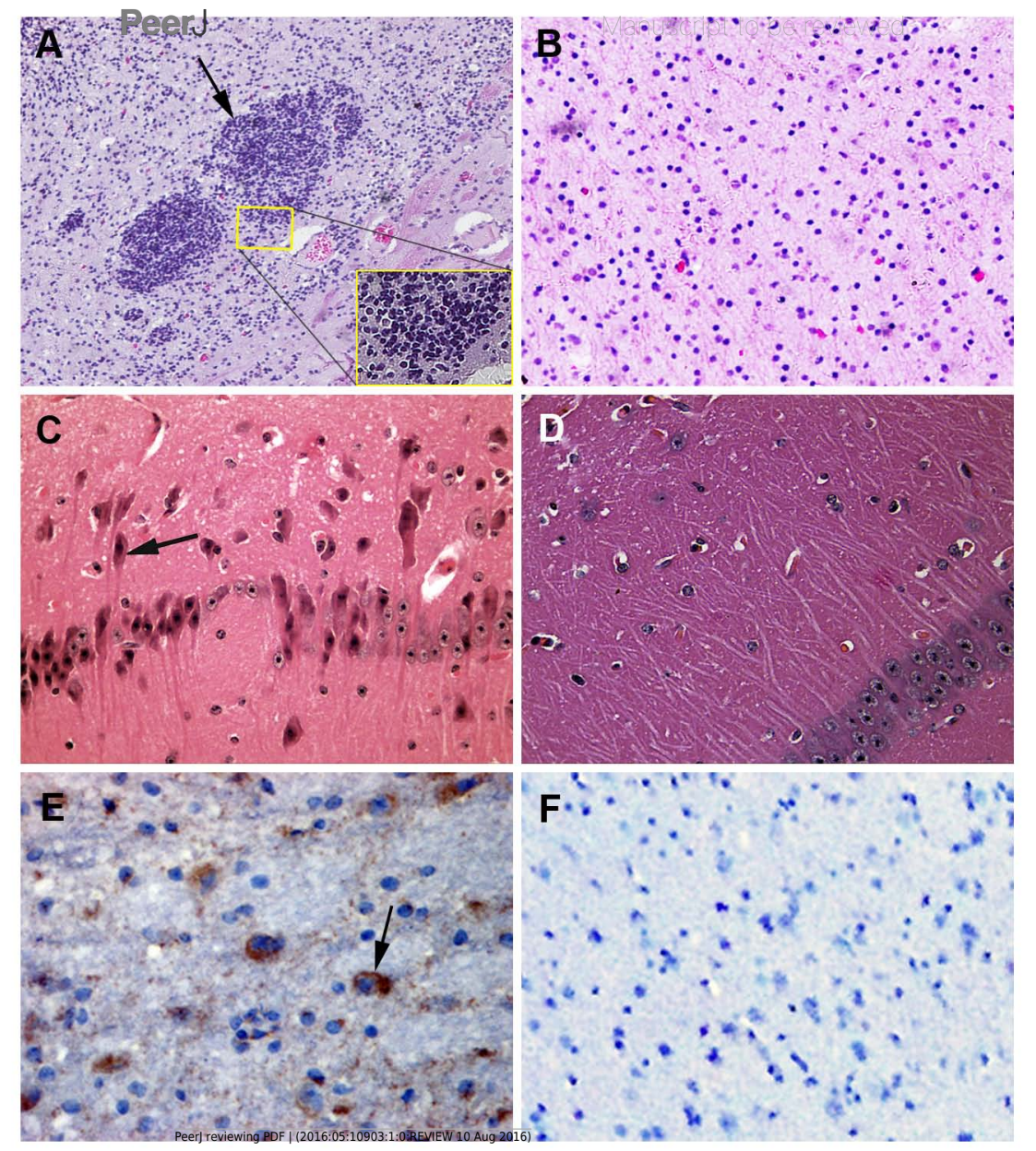


Figure 3 (on next page)

Mice experimentally infected with PHEV-CC14.

Fig.3 Mice experimentally infected with PHEV-CC14. (A) PHEV-CC14-infected mice showed arched waists, standing and vellicating front claws after at $3 \mathrm{dpi}$. (B) Mice in the control group survived normally. (C) Survival curves of BALB/C mice. $n=10$ mice per group; three independent experiments. 
Figure 4 (on next page)

Visualization of PHEV-CC14-infected brains by IF assay

Fig.4 Visualization of PHEV-CC14-infected brains from BALB/c mice by immunofluorescent assay using PHEV monoclonal antibody (diluted 1:500). Immunofluorescent assay in the cerebral cortex, showing large numbers of PHEV-positive neurons (red); original magnification, $\times 100$. PHEV-positive Purkinje cells of the cerebellum were distributed widely (red); original magnification, $\times 400$. 


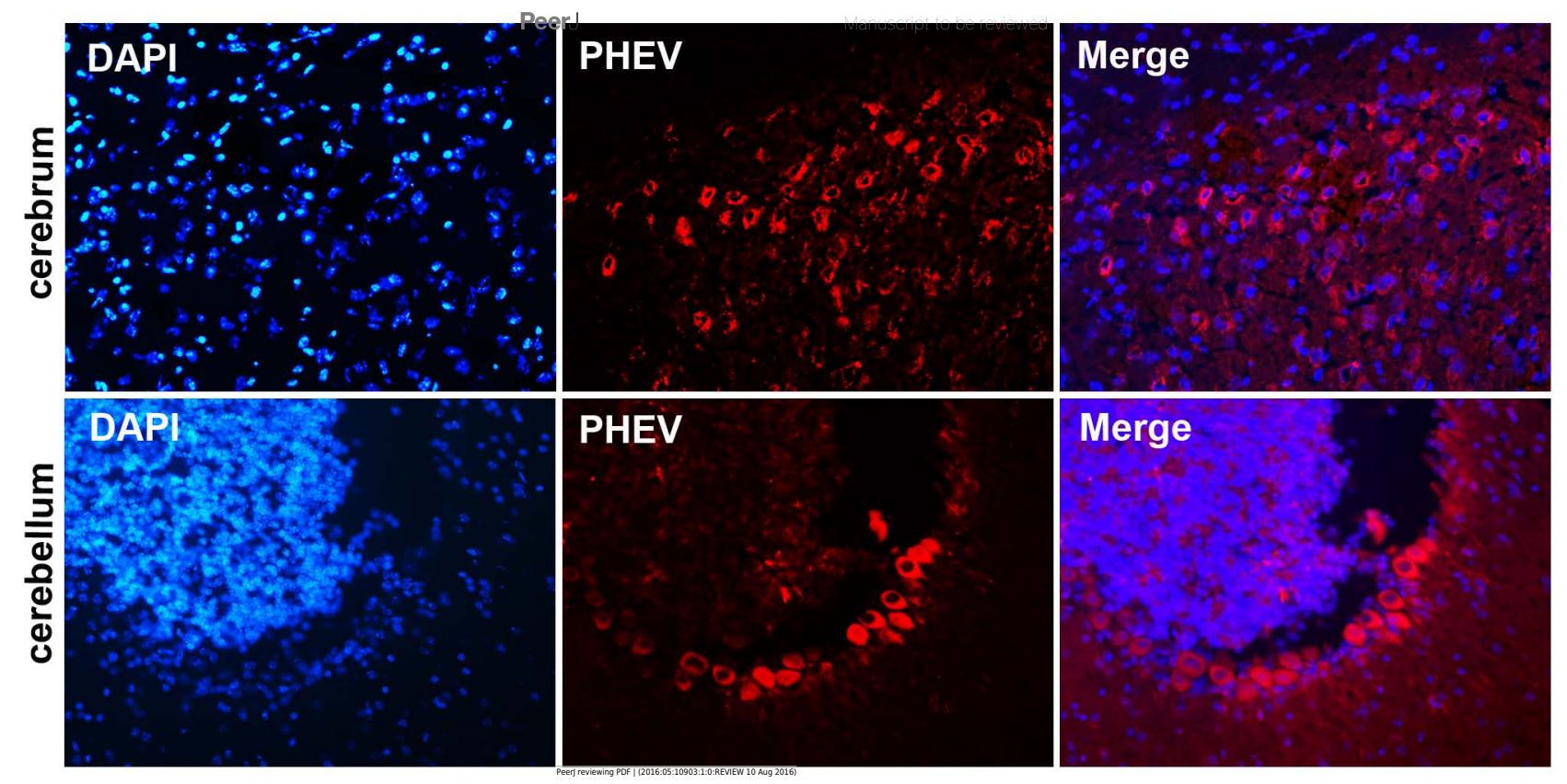




\section{Figure $\mathbf{5}$ (on next page)}

Isolation and propagation of PHEV in Neuro-2a cells.

Fig.5 Isolation and propagation of PHEV in Neuro-2a cells. (A) Mock-inoculated Neuro-2a cells showing normal cells at $4 \mathrm{dpi}, \times 200$. (B) CPE of PHEV-CC14-inoculated Neuro-2a cells at $4 \mathrm{dpi}$, showing rounded and clustered cells, $\times 200$. (C) Detection of PHEV isolate in Neuro-2a cells by immunofluorescent staining using PHEV monoclonal antibody (diluted 1:500), showing immunofluorescent-positive staining mainly evident in the cytoplasm of infected cells ( red), $\times 400$. (D) EM of PHEV-CC14-inoculated Neuro-2a cells. Crown-shaped spikes are visible. The samples were negatively stained with $3 \%$ phosphotungstic acid. The magnification bar in the picture represents $100 \mathrm{~nm}$ in length. 


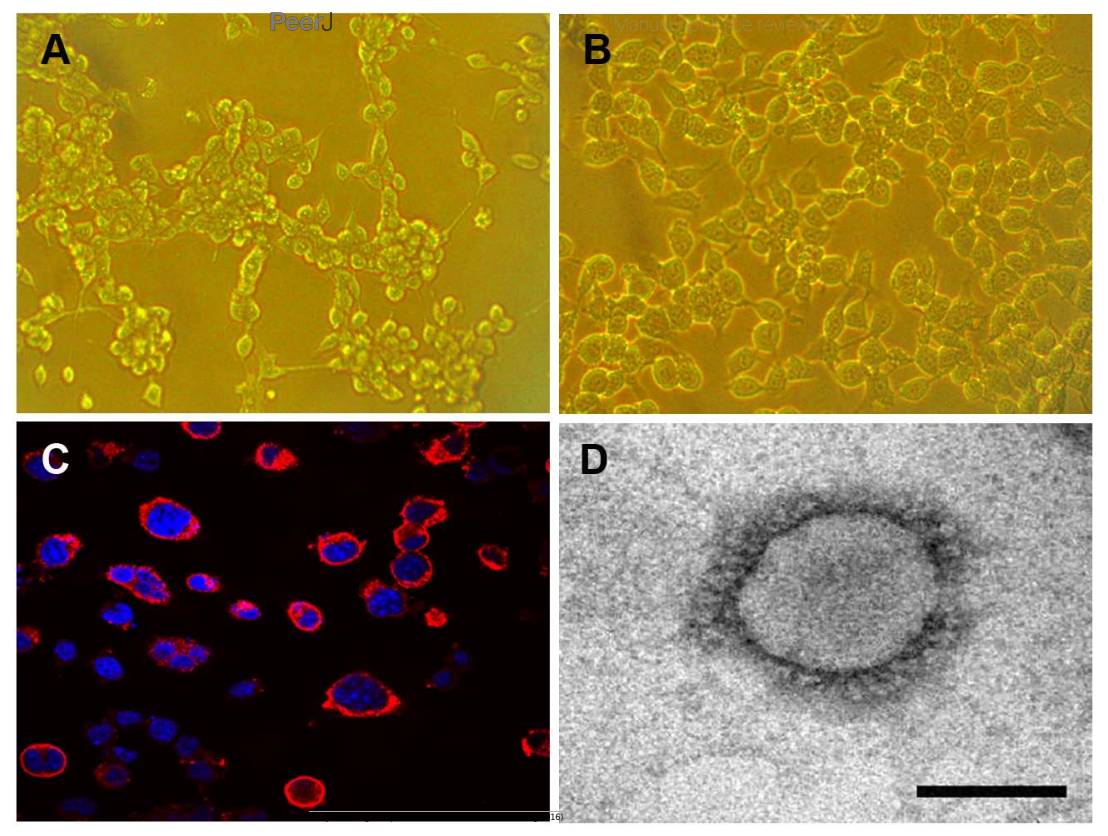


Figure $\mathbf{6}$ (on next page)

The growth curves of PHEV strains.

Fig. 6 The growth curves of PHEV strains. Neuro-2a cells were, respectively, inoculated with PHEV-CC14 and HEV 67N. The TCID ${ }_{50}$ was measured at different time points, and the growth curves were plotted. There was no significant difference in replication or proliferation between the PHEV-CC14 strain and HEV-67N strain $(p>0.05)$. 


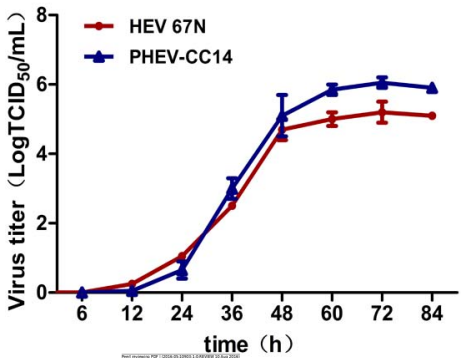




\section{Figure 7 (on next page)}

Protein structure prediction and phylogenetic analysis.

Fig.7 Protein structure prediction and phylogenetic analysis. (A) Schematic illustration of the organization of the targeted genes coding for the five structural proteins, consisting of PHEV $H E, S, E, M$ and $N$ genes (reference virus HEV-67N). (B) Phylogenetic analyses based on amino acid sequences of the five major structural proteins from PHEV in this study (indicated with triangle) and other published PHEV sequences, as well as related coronaviruses. Reference sequences obtained from GenBank are indicated by strain names and accession numbers. The trees were constructed using the neighbor-joining method in MEGA software, version 6.06. Bootstrap analysis was performed on 1,000 trials, and values are indicated adjacent to the branching points. 
A

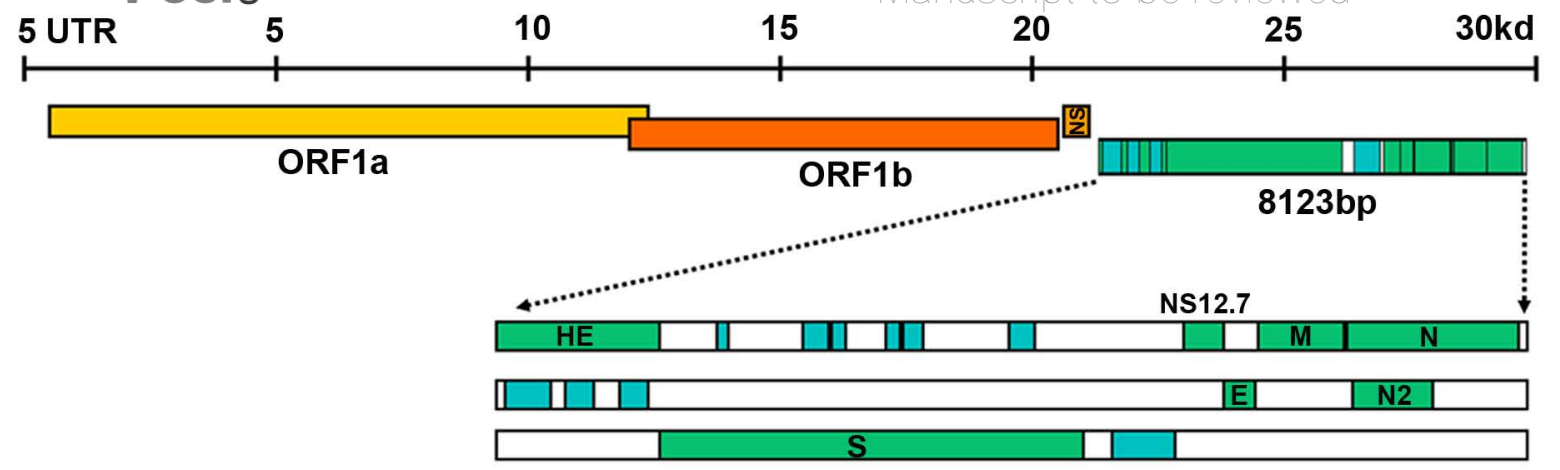

B

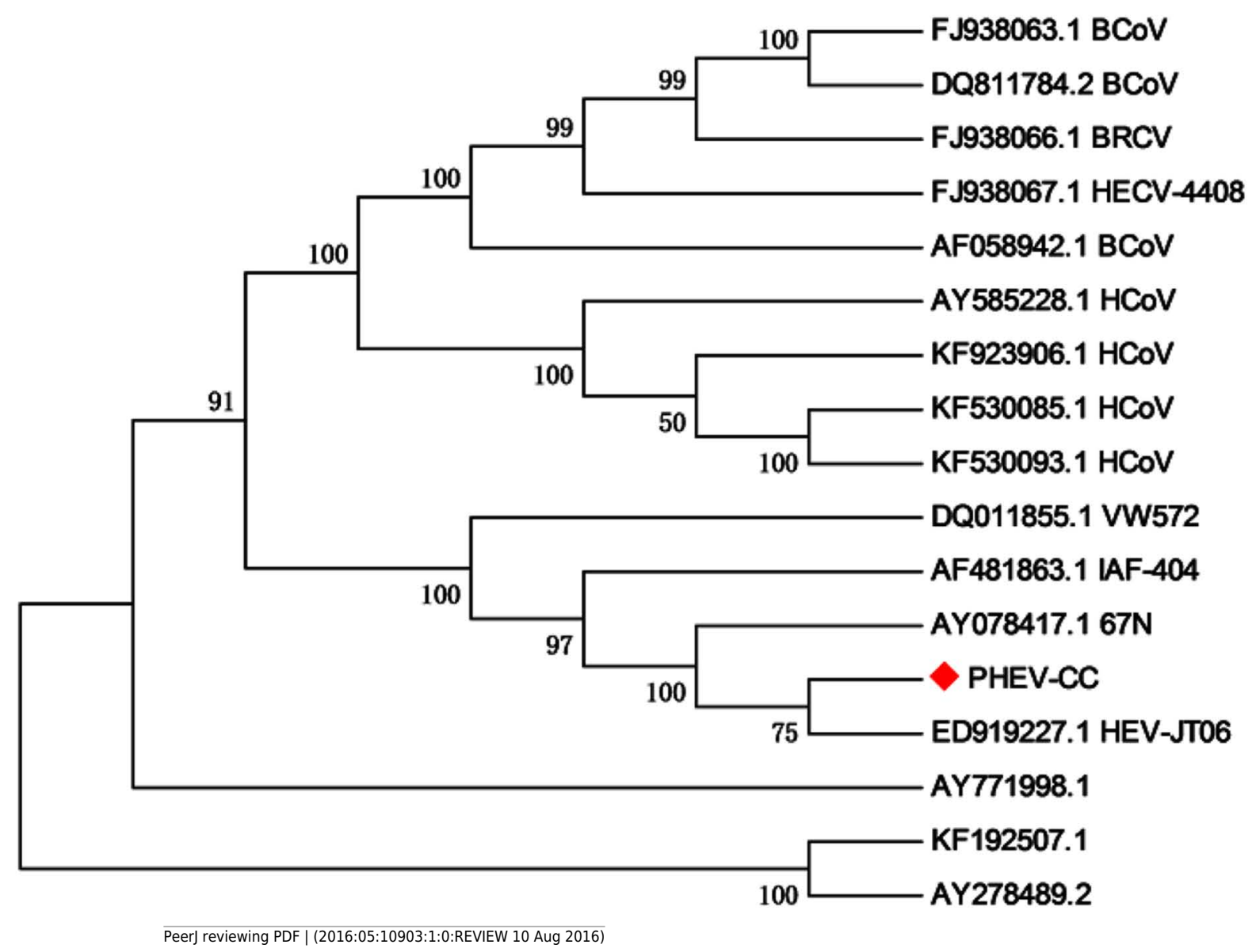


Table $\mathbf{1}$ (on next page)

Primer sets used for RT- PCR to differential diagnosis. 
1 Table 1 Primer sets used for RT-PCR to differential diagnosis

\begin{tabular}{|c|c|c|c|c|c|}
\hline Virus & Primers Sequence $\left(5^{\prime}-3^{\prime}\right)$ & $\begin{array}{l}\text { GenBank } \\
\text { No, }\end{array}$ & $\mathbf{T m}$ & Gene & $\begin{array}{l}\text { Fragment } \\
\quad \text { (bp) }\end{array}$ \\
\hline PHEV & $\begin{array}{l}\text { TACTGAAACCATTACCACT } \\
\text { CTATAACTATGACCGCGAC }\end{array}$ & AY078417.1 & 56 & $\mathrm{HE}$ & 509 \\
\hline PEDV & $\begin{array}{l}\text { GAAATAACCAGGGTCGTGGA } \\
\text { GCTCACGAACAGCCACA }\end{array}$ & DQ355221.1 & 55.3 & $\mathrm{~N}$ & 492 \\
\hline TEGV & $\begin{array}{l}\text { GATGGCGACCAGATAGAAGT } \\
\text { GCAATAGGGTTGCTTGTACC }\end{array}$ & AF302264.1 & 58 & $\mathrm{~N}$ & 612 \\
\hline DPCoV & $\begin{array}{l}\text { CGCGTAATCGTGTGATCTATGT } \\
\text { CCGGCCTTTGAAGTGGTTAT }\end{array}$ & KJ569769 & 57.4 & $\mathrm{M}$ & 541 \\
\hline PRV & $\begin{array}{c}\text { CCGGCCTTTGAAGTGGTTAT } \\
\text { CGACCTGGCGTTTATTAACCGAGA }\end{array}$ & M61196.1 & 56 & $\mathrm{gH}$ & 355 \\
\hline
\end{tabular}

2 


\section{Table 2 (on next page)}

RT-PCR detection of PHEV and other relevant viruses on tissues. 
1 Table 2 RT-PCR detection of PHEV and other relevant porcine viruses on tissue samples

2 from nine pigs in Jilin province, China

\begin{tabular}{ccccccc}
\hline \multirow{2}{*}{ Pig age } & Original & \multicolumn{5}{c}{ PHEV and relevant porcine virus detection } \\
\cline { 2 - 6 } & samples & PHEV & PEDV & TEGV & DPCoV & PRV \\
\cline { 2 - 6 } & brain & 6 & - & - & - & - \\
\multirow{2}{*}{ 1-week old } & spinal cord & 6 & - & - & - & - \\
& IC a & 4 & - & - & - & - \\
& spleen & - & - & - & - & - \\
& kidneys & - & - & - & - & - \\
& lungs & - & - & - & - & - \\
\hline \multirow{3}{*}{$<3-w e e k$ old } & brain & 2 & - & - & - & - \\
& spinal cord & 2 & - & - & - & - \\
& IC a & - & - & - & - & - \\
& spleen & - & - & - & - & - \\
& kidneys & - & - & - & - & - \\
& lungs & - & - & - & - & - \\
\hline
\end{tabular}

${ }^{\mathrm{a}} \mathrm{IC}$, intestinal contents. 


\section{Table 3 (on next page)}

Sequences of the oligonucleotides for gene-walking RT-PCR. 
1 Table 3 Sequences of the oligonucleotides for gene-walking RT-PCR

\begin{tabular}{|c|c|c|c|}
\hline Primers & Primers Sequence $\left(5^{\prime}-3^{\prime}\right)$ & Gene & Fragment (bp) \\
\hline $\mathrm{P} 1 / \mathrm{P} 2$ & $\begin{array}{l}\text { TACTGAAACCATTACCACT } \\
\text { CTATAACTATGACCGCGAC }\end{array}$ & I & 1275 \\
\hline $\mathrm{P} 3 / \mathrm{P} 4$ & $\begin{array}{l}\text { GAAATAACCAGGGTCGTGGA } \\
\text { GCTCACGAACAGCCACA }\end{array}$ & II & 1614 \\
\hline P5/P6 & $\begin{array}{l}\text { GATGGCGACCAGATAGAAGT } \\
\text { GCAATAGGGTTGCTTGTACC }\end{array}$ & III & 1674 \\
\hline $\mathrm{P} 7 / \mathrm{P} 8$ & $\begin{array}{c}\text { CGCGTAATCGTGTGATCTATGT } \\
\text { CCGGCCTTTGAAGTGGTTAT }\end{array}$ & IV & 1390 \\
\hline P9/P10 & $\begin{array}{c}\text { CCGGCCTTTGAAGTGGTTAT } \\
\text { CGACCTGGCGTTTATTAACCGAGA }\end{array}$ & V & 256 \\
\hline P11/P12 & $\begin{array}{c}\text { ATGAGTAGTCCAACTACAC } \\
\text { TATTTCTCAACAATGCGGTGTC }\end{array}$ & VI & 685 \\
\hline $\mathrm{P} 13 / \mathrm{P} 14$ & $\begin{array}{l}\text { TCAGGCATGGACACCGCATT } \\
\text { AGAGTGCCTTATCCCGACTTT }\end{array}$ & VII & 1463 \\
\hline $\mathrm{P} 15 / \mathrm{P} 16$ & $\begin{array}{l}\text { TTACAGCACTTAGATCACGTAGAT } \\
\text { TAAACTCTGGCTTCGCCAGGTAAT }\end{array}$ & VIII & 2195 \\
\hline
\end{tabular}

2 